\title{
Symmetry and Correlation Effects on Band Structure Explain the Anomalous Transport Properties of (111) $\mathrm{LaAlO}_{3} / \mathrm{SrTiO}_{3}$
}

\author{
Udit Khanna, ${ }^{1}$ P. K. Rout, ${ }^{1}$ Michael Mograbi, ${ }^{1}$ Gal Tuvia, ${ }^{1}$ Inge Leermakers, ${ }^{2}$ Uli Zeitler, ${ }^{2}$ \\ Yoram Dagan, ${ }^{1}$ and Moshe Goldstein ${ }^{1}$ \\ ${ }^{1}$ Raymond and Beverly Sackler School of Physics and Astronomy, Tel-Aviv University, Tel Aviv, 6997801, Israel \\ ${ }^{2}$ High Field Magnet Laboratory (HFML-EFML), Radboud University, 6525 ED Nijmegen, Netherlands
}

(Received 30 January 2019; published 17 July 2019)

\begin{abstract}
The interface between the two insulating oxides $\mathrm{SrTiO}_{3}$ and $\mathrm{LaAlO}_{3}$ gives rise to a two-dimensional electron system with intriguing transport phenomena, including superconductivity, which are controllable by a gate. Previous measurements on the (001) interface have shown that the superconducting critical temperature, the Hall density, and the frequency of quantum oscillations, vary nonmonotonically and in a correlated fashion with the gate voltage. In this Letter we experimentally demonstrate that the (111) interface features a qualitatively distinct behavior, in which the frequency of Shubnikov-de Haas oscillations changes monotonically, while the variation of other properties is nonmonotonic albeit uncorrelated. We develop a theoretical model, incorporating the different symmetries of these interfaces as well as electronic-correlation-induced band competition. We show that the latter dominates at (001), leading to similar nonmonotonicity in all observables, while the former is more important at (111), giving rise to highly curved Fermi contours, and accounting for all its anomalous transport measurements.
\end{abstract}

DOI: 10.1103/PhysRevLett.123.036805

Introduction.-The high-mobility two-dimensional electron system (2DES) at the interface of $\mathrm{SrTiO}_{3}$ and $\mathrm{LaAlO}_{3}$ [1] shows a variety of quantum transport phenomena [2-6], in addition to a rich phase diagram including magnetism [7-9] and superconductivity [10-12] at low temperatures. The multiorbital band structure of the system, which gives rise to this physics, has been the subject of many studies. The electronic structure of the interface has been probed via optical methods such as x-ray absorption spectroscopy $[13,14]$ and angle resolved photoemission spectroscopy $[15,16]$ as well as through magnetotransport $[5,17-20]$, which were supplemented by density functional theory based ab initio calculations [21-25] and analytical studies [26,27]. Most studies concentrated on the (001) interface, although a conducting 2DES can arise in other interfaces [28]. This has changed recently with several works [29-44] indicating that the (111) interface has a distinct electronic structure with novel properties.

To elucidate the electronic properties of (111) $\mathrm{LaAlO}_{3} / \mathrm{SrTiO}_{3}$, we embarked on a combined experimental and theoretical study. Experimentally we focus on magnetotransport at the (111) interface (Hall effect, quantum oscillations, and superconductivity), which shows surprising differences from the (001) interface [5]: In (001) all these quantities are nonmonotonic and reach their maximum at roughly the same gate voltage, whereas at (111) the quantum oscillation frequency is monotonic, and the peaks in the Hall density and superconducting transition temperature are well separated. To understand these results, we calculate the correlation-induced band structure of the 2DES, taking into account the crystal structure and the change in symmetry from the bulk (octahedral) to the interface [triangular in (111), square in (001)] [42-44]. We elucidate the different behavior of the (111) as compared to the (001) interface: While the latter is dominated by interaction-induced population transfer, the former is governed by symmetry-induced Fermi contour shape. The resulting transport coefficients nicely follow the experimental data.

Transport measurements. - 14 monolayer thick epitaxial thin films of $\mathrm{LaAlO}_{3}$ were grown on an atomically flat Ti-terminated $\mathrm{SrTiO}_{3}$ (111) substrate using the pulsed laser deposition technique in combination with reflection high energy electron diffraction. Details of the deposition procedure and substrate treatment are described in Ref. [32]. Electrical transport measurements of the $80 \mu \mathrm{m} \times 260 \mu \mathrm{m}$ Hall bar, patterned along the [12̄1] direction using optical lithography [32], were performed by a four probe ac technique with a current of $50 \mathrm{nA}$ in a custom made ${ }^{3} \mathrm{He}$ cryostat equipped with a $35 \mathrm{~T}$ magnet.

We investigated magnetotransport at the (111) interface under a perpendicular field to understand the behavior of the carrier density $\left(n_{2 \mathrm{D}}\right)$ as a function of temperature $(T)$ and gate voltage $\left(V_{g}\right)$ in a back-gated device. $n_{2 \mathrm{D}}$ was extracted using both the Hall density $\left[n_{\mathrm{Hall}}=\left(e R_{H}\right)^{-1}\right.$, where $R_{H}$ is the slope of the low-field Hall resistivity] and the Shubnikov-de Haas $(\mathrm{SdH})$ oscillations (through the Onsager relation [45]) observed at higher magnetic fields. 


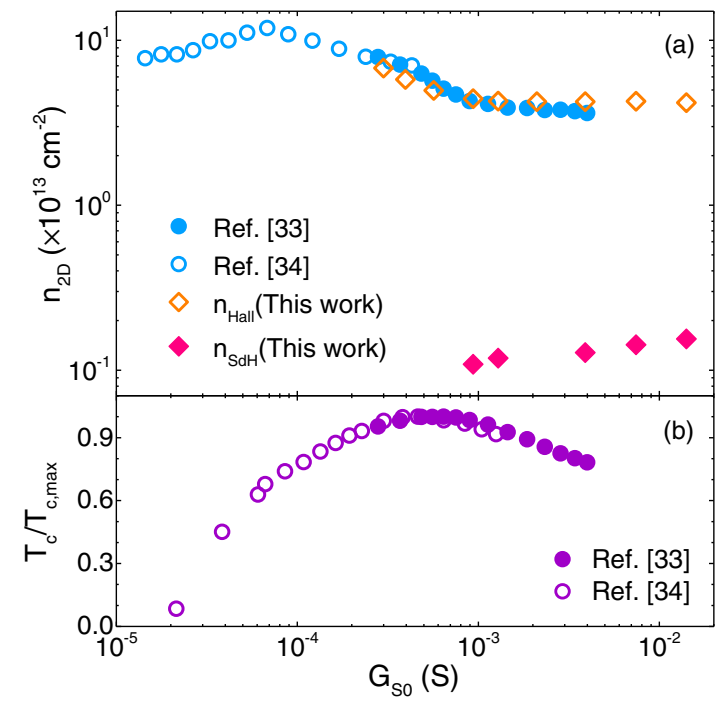

FIG. 1. Gate dependence of transport parameters. (a) The sheet carrier density $\left(n_{2 \mathrm{D}}\right)$ determined from low-field Hall measurements and quantum oscillations as a function of the zero field sheet conductance $\left(G_{S 0}\right)$. (b) Normalized superconducting critical temperature $T_{c} / T_{\mathrm{c}, \max }$ as a function of $G_{S 0}$.

We also studied corresponding variation of the superconducting transition temperature. The back gate was employed to control the carrier density and vary the sheet conductance $\left(G_{S}\right)$. Since the gate response changes between different sample cooldowns and $V_{g}$ sweeps, we present the results in Fig. 1 as a function of the zero field conductance $G_{S 0}$ [34]. The $V_{g}$ dependence can be found in Ref. [45].

Figure 1(a) compares the variation of carrier density from the $\mathrm{SdH}$ analysis $\left(n_{\mathrm{SdH}}\right)$ and the Hall measurement $\left(n_{\text {Hall }}\right)$ with the gate voltage $\left(V_{g}\right)$, while Fig. 1(b) presents the corresponding dependence of the superconducting critical temperature $\left(T_{c}\right)$. The observed variation and values of $n_{\text {Hall }}$ are consistent with our previous results on the (111) interface $[33,34]$ [also shown in Fig. 1(a)] and with other recent results $[35,36]$.

Curiously, we find that while $n_{\text {Hall }}$ and $T_{c}$ are nonmonotonic functions of $V_{g}, n_{\mathrm{SdH}}$ changes monotonically. Moreover, the peak in $n_{\text {Hall }}$ appears when quantum oscillations are not observable. These features are strikingly different from our previous measurements on the (001) interface [5]. In the latter case, $n_{\mathrm{SdH}}$ also changes nonmonotonically with $V_{g}$ and the maximal $n_{\mathrm{SdH}}, n_{\mathrm{Hall}}$, and $T_{c}$ appear at roughly the same gate voltage.

At both interfaces $n_{\mathrm{SdH}}$ is much smaller than $n_{\mathrm{Hall}}$. Since the $\mathrm{SdH}$ signal decays exponentially with inverse scattering time, this indicates the presence of two low-energy bands in the electronic structure with different mobilities. Therefore, both bands would contribute to $n_{\text {Hall }}$ but only the mobile one would be observable through the quantum oscillation measurements.

We note that the band structure of (111) $\mathrm{LaAlO}_{3} / \mathrm{SrTiO}_{3}$ has recently been probed using Hall measurements [36].
However, the Hall coefficient receives contributions from all the bands and also depends on the corresponding scattering times, making it hard to decipher the band structure. The crucial new ingredient here is the quantum oscillations, which directly probe the population of the more mobile band, and demonstrate the qualitative difference between the (111) and (001) interfaces. These allow us to develop a complete and consistent theoretical picture for both interfaces, as we now describe.

Theoretical model.-We first consider the orbital character of the relevant levels at the two interfaces. Ab initio studies [21-23] show that the low energy conduction bands in bulk $\mathrm{SrTiO}_{3}$ are composed of the $t_{2 g}$ orbitals of the $\mathrm{Ti}$ atoms. These are degenerate in the bulk due to their cubic arrangement (the low temperature structural distortions are negligible for our purposes), which imparts octahedral symmetry to the band structure. However, the reduced symmetry at the interfaces can lift the degeneracy and modify the orbital character.

At the (001) interface, Ti atoms form a square lattice, which does not modify the in-plane crystal field. In combination with the confining potential, the degeneracy of the $t_{2 g}$ orbitals is lifted but the orbital character is not modified. Specifically, if the confinement is along the $z$ direction, then the $x y$ orbital is lowered in energy due to its higher effective mass in the confinement direction [21] [Fig. 2(d)]. On the other hand, at the (111) interface, Ti atoms form a stacked triangular lattice with three interlaced
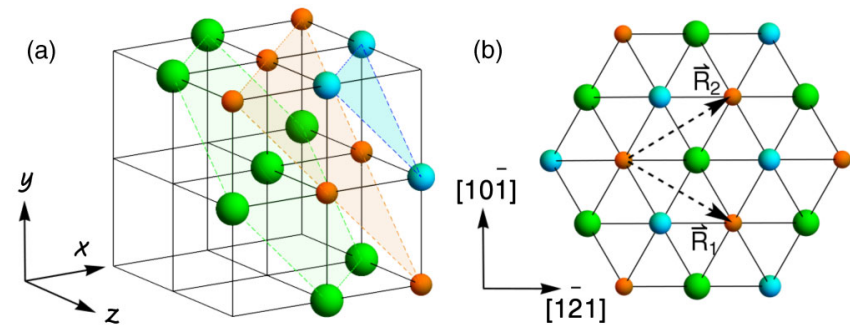

(c)

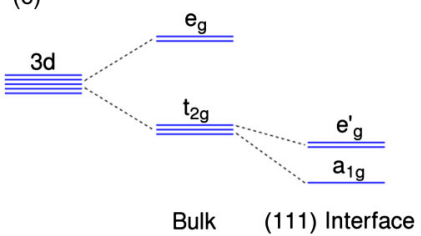

(d)

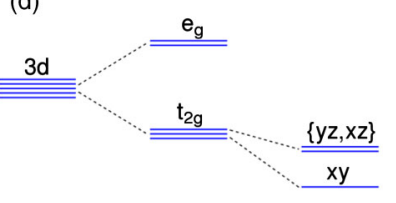

Bulk (001) Interface
FIG. 2. Crystal structure of the (111) $\mathrm{LaAlO}_{3} / \mathrm{SrTiO}_{3}$ interface. (a) Three inequivalent layers of $\mathrm{Ti}$ atoms (green $\equiv \mathrm{Ti}_{1}$, orange $\equiv$ $\mathrm{Ti}_{2}$, blue $\equiv \mathrm{Ti}_{3}$ ) forming one unit cell at the (111) interface of a cubic lattice. (b) Top view of the (111) trilayer. $\vec{R}_{1,2}$ are the lattice vectors of the triangular lattice at the interface. (c),(d) Level structure of the $\mathrm{Ti} 3 d$ orbitals at the (111) and (001) interfaces, respectively. The bulk cubic structure leads to the splitting into $t_{2 g}$ and $e_{g}$ orbitals. (c) The trigonal crystal symmetry at the (111) interface leads to further splitting of $t_{2 g}$ into $a_{1 g}$ and $e_{g}^{\prime}$ orbitals. (d) The in-plane crystal symmetry does not change at the (001) interface but the surface confinement lifts the degeneracy. 
layers [Figs. 2(a), 2(b)]. This changes the bulk octahedral symmetry to triangular at the interface and introduces a new in-plane crystal field [50], which hybridizes the $t_{2 g}$ orbitals, forming $\left|a_{1 q}\right\rangle=(|x y\rangle+|y z\rangle+|x z\rangle) / \sqrt{3}$ and $\left|e_{q \pm}^{\prime}\right\rangle=\left(|x y\rangle+\omega^{ \pm 1}|y z\rangle+\omega^{ \pm 2}|x z\rangle\right) / \sqrt{3}$, where $\omega=e^{2 \pi i / 3}$. Their splitting is sensitive to details of the interface. Here, we choose parameters such that $a_{1 g}$ is lower in energy [Fig. 2(c)], in accordance with recent XLD experiments [44] and DFT calculations $[42,43]$.

Next, we employ a tight-binding model with these orbitals on the first three inequivalent layers [Fig. 2(a)], keeping track of the separation and connectivity of sites on the different layers [51]. In the basis, $\left\{\left|a_{1 g}\right\rangle,\left|e_{g+}^{\prime}\right\rangle,\left|e_{g-}^{\prime}\right\rangle\right\} \otimes\left\{\left|\mathrm{Ti}_{1}\right\rangle,\left|\mathrm{Ti}_{2}\right\rangle,\left|\mathrm{Ti}_{3}\right\rangle\right\} \otimes\{|\uparrow\rangle,|\downarrow\rangle\}$, the hopping terms can be written as $18 \times 18$ matrices given by

$$
H_{0}^{(111)}(\vec{K})=\left(\begin{array}{ccc}
A(\vec{K}) & B(\vec{K}) & B^{\dagger}(\vec{K}) \\
B^{\dagger}(\vec{K}) & A(\vec{K}) & B(\vec{K}) \\
B(\vec{K}) & B^{\dagger}(\vec{K}) & A(\vec{K})
\end{array}\right) \otimes \mathcal{I}_{2},
$$

where the block matrices $A(\vec{K})$ and $B(\vec{K})$ are

$$
\begin{gathered}
A(\vec{K})=-\frac{\left(2 t+t^{\prime}\right)}{3}\left(\begin{array}{ccc}
0 & e^{-i K_{2}} f_{0}(\vec{K}) & 0 \\
e^{i K_{2}} f_{0}(-\vec{K}) & 0 & e^{-i K_{1}} f_{0}(\vec{K}) \\
0 & e^{i K_{1}} f_{0}(-\vec{K}) & 0
\end{array}\right)-\frac{t^{\prime \prime}}{3}\left(\begin{array}{ccc}
2 \epsilon_{0}(\vec{K}) & 0 & f_{0}(-\vec{K}) \\
0 & 2 \epsilon_{0}(\vec{K}) & 0 \\
f_{0}(\vec{K}) & 0 & 2 \epsilon_{0}(\vec{K})
\end{array}\right), \\
B(\vec{K})=\omega^{2} \frac{\left(t-t^{\prime}\right)}{3}\left(\begin{array}{ccc}
0 & e^{-i K_{2}} f_{\omega}(\vec{K}) & 0 \\
e^{i K_{2}} f_{\omega}(-\vec{K}) & 0 & e^{-i K_{1}} f_{\omega}(\vec{K}) \\
0 & e^{i K_{1}} f_{\omega}(-\vec{K}) & 0
\end{array}\right)-\omega^{2} \frac{t^{\prime \prime}}{3}\left(\begin{array}{ccc}
2 \epsilon_{\omega}(\vec{K}) & 0 & f_{\omega}(-\vec{K}) \\
0 & 2 \epsilon_{\omega}(\vec{K}) & 0 \\
f_{\omega}(\vec{K}) & 0 & 2 \epsilon_{\omega}(\vec{K})
\end{array}\right),
\end{gathered}
$$

where, $t$ and $t^{\prime}$ are the light and heavy nearest-neighbor hopping amplitudes while $t^{\prime \prime}$ is the next-nearest-neighbor hopping. $f_{0}(\vec{K})=1+e^{i K_{1}}+e^{i K_{2}}, f_{\omega}(\vec{K})=1+\omega e^{i K_{1}}+$ $\omega^{2} e^{i K_{2}}, \epsilon_{0}(\vec{K})=\cos \left(K_{1}\right)+\cos \left(K_{2}\right)+\cos \left(K_{1}-K_{2}\right)$, and $\epsilon_{\omega}(\vec{K})=\cos \left(K_{1}-K_{2}\right)+\omega \cos \left(K_{2}\right)+\omega^{2} \cos \left(K_{1}\right)$, where $K_{1,2} \in[-\pi, \pi]$, the two-dimensional Brillouin zone. The atomic spin-orbit coupling is an on-site term mixing the orbitals and spin states. Taking the spin quantization axis along the (111) direction, the spin-orbit coupling is

$$
H_{\mathrm{so}}^{(111)}=\frac{\Delta_{\mathrm{so}}}{2}\left(\begin{array}{ccc}
0 & -\sqrt{2} \sigma^{+} & \sqrt{2} \sigma^{-} \\
-\sqrt{2} \sigma^{-} & -\sigma_{z} & 0 \\
\sqrt{2} \sigma^{+} & 0 & \sigma_{z}
\end{array}\right) \text {, }
$$

where $\sigma^{ \pm}=\left(\sigma_{x} \pm i \sigma_{y}\right) / 2$, with $\sigma_{x, y, z}$ being the Pauli matrices. Additionally, the single-particle Hamiltonian includes the trigonal crystal-field $\Delta_{\mathrm{cf}}$ (which lifts the degeneracy between the orbitals) and a linear confining potential $V_{c}$ (which lifts the layer degeneracy) [45].

Finally, correlation effects are incorporated through an on-site Hubbard term $\sum_{r} \sum_{I \neq J} U n_{r I} n_{r J}$, which includes both interorbital and intra-orbital repulsion (assumed to be of equal strength in order to reduce the number of free parameters). The two-body term is then treated in the Hartree-Fock approximation. The mean-field ansatz is that the ground state is invariant under time reversal and has the full symmetry of the (interface) crystal structure, i.e., the $C_{3 v}$ group at the (111) interface (we have verified that tetragonal distortions, etc., have a small effect on our results). Under this assumption, the Hubbard term reduces to a one-body term with four independent real parameters (per layer) - the occupancy of the three orbitals (which appear in the Hartree terms) and one spin-mixing average (Fock term) which renormalizes the spin-orbit interaction. We note that a state with the full crystal symmetry must have equal occupancy of the $x y, y z$, and $x z$ orbitals. Therefore, in terms of the original $t_{2 g}$ orbitals, there is only one independent Hartree term and three Fock terms.

The mean-field Hamiltonian is solved self-consistently, using $t=V_{c}=437.5 \mathrm{meV}, t^{\prime}=t^{\prime \prime}=20 \mathrm{meV}, \Delta_{\text {so }}=3 \mathrm{meV}$, $\Delta_{\mathrm{cf}}=2 \mathrm{meV}$ and $U=2 \mathrm{eV}$. These parameter values are close to those employed previously for the (001) interface [5,17-19]. Although surface reconstruction can lead to different parameters at the two interfaces, the qualitative behavior is not expected to change.

Theoretical results.-Figure 3 shows the results of the self-consistent calculation for the (111) interface. Figures 3(a), 3(b) show the dispersion of the two lowest energy bands at two different chemical potentials $(\mu)$ and Figs. 3(c), 3(d) show the corresponding Fermi contours. For small values of $\mu$, only the lowest band is occupied. Close to the $\Gamma$ point, it mostly consists of the $a_{1 g}$ orbital. Away from $\Gamma$, the orbital character changes and becomes anisotropic. At larger $\mu$, the second band is also populated 

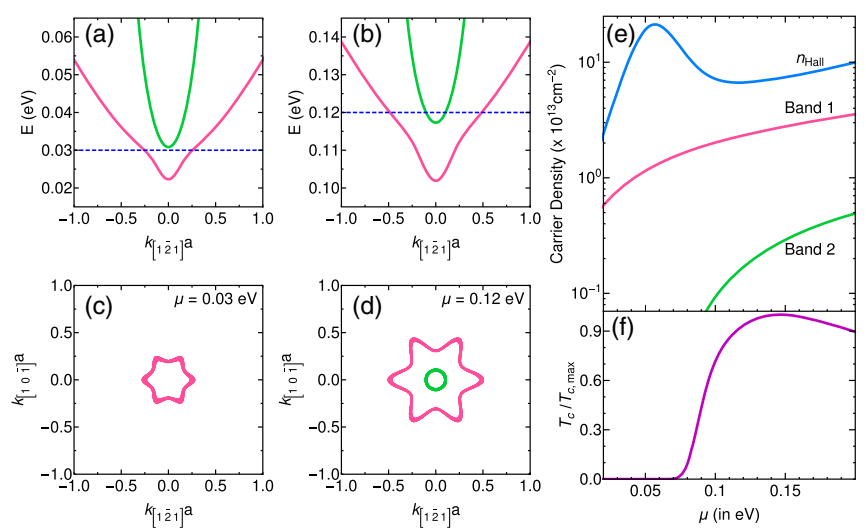

FIG. 3. Results of the theoretical model for the (111) interface. (a), (b) Band structure for two different chemical potentials $\mu$ before $(\mu=0.03 \mathrm{eV})$ and after $(\mu=0.12 \mathrm{eV})$ the second band starts getting occupied. The pink (green) line corresponds to band 1 (2), which is composed of $a_{1 g}\left(e_{g}^{\prime}\right)$ orbitals close the $\Gamma$ point. The dashed blue line marks the Fermi energy. (c), (d) The Fermi contours corresponding to the band structures in (a), (b), respectively. The outer surface (corresponding to band 1) is highly anisotropic at all $\mu$. (e) The carrier and Hall densities as a function of $\mu$. The carrier density is monotonic for both bands, while the Hall density is nonmonotonic. The peak in $n_{\mathrm{Hall}}$ occurs before the second band starts getting populated. This is in accordance with the experiment (Fig. 1) and indicates that it is due to the anisotropic shape of the lowest band. (f) Normalized superconducting critical temperature $\left(T_{c} / T_{\mathrm{c}, \max }\right)$ as a function of $\mu$ within the single-band BCS model.

[Figs. 3(b), 3(d)]. For the range of $\mu$ relevant here, this band consists primarily of one of the $e_{g}^{\prime}$ orbitals and remains almost parabolic. Crucially, Fig. 3(e) shows the monotonic variation of carrier density of the two bands as a function of $\mu$. The monotonic rise of the second band population agrees quite well with the SdH data [Fig. 1(a)], and supports our assumption that only this band gives rise to visible quantum oscillations, due to its higher mobility.

Upon increasing gate voltage the measured $n_{\text {Hall }}$ [Fig. 1(a)] has a peak before the quantum oscillations are visible. This means that this observed nonmonotonicity must arise from the lowest band by itself. This is an important difference between the (001) and the (111) interfaces that can be identified here because of our combination of $\mathrm{SdH}$ and Hall measurements. Figures 3(c),3(d) show that the first band is nonparabolic and consists of regions with both positive and negative curvature, throughout the range of relevant chemical potential. This implies that a wave packet gliding around the constant energy surface will give both electronlike and holelike contributions to the Hall conductivity. This is further complicated by the momentum-dependent orbital character of the band at large filling. Under these conditions, the standard Drude relation between inverse Hall coefficient and the carrier density of a single band $\left[n_{b}=\left(e R_{H}\right)^{-1}\right]$ is no longer valid and $n_{\text {Hall }}$ can differ significantly from the actual band population. Similarly, the two band model, often used to fit Hall data for oxide interfaces, is valid only in the case of two isotropic bands with no orbital mixing and therefore is not directly applicable for (111) $\mathrm{LaAlO}_{3} / \mathrm{SrTiO}_{3}$.

To properly account for these features we compute the longitudinal and Hall conductivity $\left(\sigma_{L}\right.$ and $\left.\sigma_{H}\right)$ using general expressions derived from the Boltzmann equation assuming momentum dependent scattering times $[45,52,53]$. Specifically, we fix the orbital lifetimes $\left(\tau_{a_{1 g}}\right.$ and $\left.\tau_{e_{g \pm}^{\prime}}\right)$ and assume the scattering time for the $m$ th band to be $\tau_{m}(\vec{K})=\sum_{\sigma} \tau_{a_{1 q}}\left|\psi_{m}\left(a_{1 g}, \sigma, \vec{K}\right)\right|^{2}+$ $\tau_{e_{g}^{\prime}}\left(\left|\psi_{m}\left(e_{g+}^{\prime}, \sigma, \vec{K}\right)\right|^{2}+\left|\psi_{m}\left(e_{g-}^{\prime}, \sigma, \vec{K}\right)\right|^{2}\right)$, where $\psi_{m}$ is the self-consistent wave function for the $m$ th band. This allows $\tau_{m}(\vec{K})$ to trace the changes in orbital character along the Fermi contours. Here we choose $\tau_{e_{g}^{\prime}}=10 \tau_{a_{1 g}}$, so that the second band is more mobile. While $\sigma_{L}$ and $\sigma_{H}$ depend on the orbital lifetimes separately, and are thus harder to constrain by experimental data, $n_{\text {Hall }}\left(\approx \sigma_{L}^{2} / \sigma_{H}\right)$ depends only on the ratio of the lifetimes. Therefore we show the variation of $n_{\text {Hall }}$ as a function of $\mu$ in Fig. 3(e). The decent agreement of this theoretical result with experimental data from Fig. 1(a) implies that the experimental observations are a consequence of the shape and orbital character of the lowest band.

We note that the Fermi contours in Figs. 3(c), 3(d) are similar to those reported for the (111) surface of $\mathrm{SrTiO}_{3}$ [30]. However, unlike the band structure in Figs. 3(a), 3(b) Ref. [30] did not observe any splitting between the two lowest bands. This difference stems from the change in order of $a_{1 g}$ and $e_{g}^{\prime}$ bands between the $\mathrm{SrTiO}_{3}$ surface and $\mathrm{LaAlO}_{3} / \mathrm{SrTiO}_{3}$ interface [42-44]. In our model, this order is fixed by the sign of $\Delta_{\mathrm{cf}}$, which we choose in accordance with Ref. [44]. Using the opposite sign would provide a band structure similar to the one reported in Ref. [30].

Figure 4 shows the results of a similar calculation for the (001) interface using a closely related model [5,19]. Figures 3(e) and 4(e) markedly differ in the behavior of the carrier density of band 2 at the two interfaces: Here the population of band $2\left(n_{\mathrm{SdH}}\right)$ is nonmonotonic, and the Hall density follows it [as opposed to monotonic $\mathrm{SdH}$ and maximal Hall number when band 2 is empty in (111)]. We stress that the nonmonotonic behavior of band 2 population at the (001) interface [Fig. 4(e)] is not due to larger interaction terms (the three largest parameters, $U, t$, and $t^{\prime}$, were taken to be equal in both cases). Rather it occurs because at the (001) interface the bands retain their original orbital characters $(x y, y z$, and $x z$ ), which have a large difference in effective mass in the interface plane. This generates a correlation-induced population transfer among the bands, because the total energy can be minimized by transferring electrons from the lighter to heavier band $[5,19,20]$. Since band 2 changes from heavy to light with increasing $\mu$ [Figs. 4(a), 4(b)], it is first populated then 

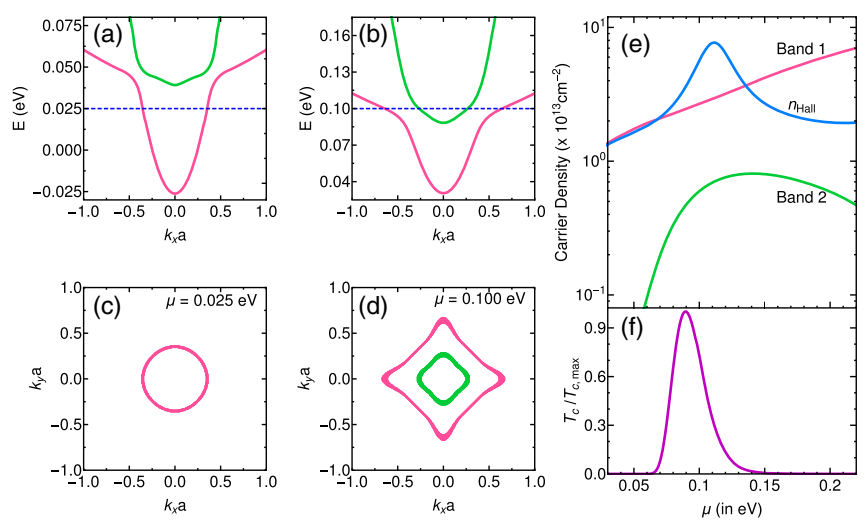

FIG. 4. Results of the theoretical model for the (001) interface. (a),(b) Band structure for two different chemical potentials $\mu$ before $(\mu=0.025 \mathrm{eV})$ and after $(\mu=0.10 \mathrm{eV})$ the second band starts getting occupied. The pink (green) line corresponds to band 1 (2), which is composed of $x y$ (other $t_{2 g}$ ) orbitals close to the $\Gamma$ point. The dashed blue line marks the Fermi energy. (c),(d) The Fermi contours corresponding to the band structures in (a),(b), respectively. The outer surface (corresponding to band 1) is isotropic at low values of $\mu$. When the second band gets populated, the orbital character of the bands switches and the outer band becomes anisotropic. (e) The carrier and Hall densities as a function of $\mu$. The carrier density for the second band is nonmonotonic due to correlation-induced transfer of electrons from the lighter to heavier band. The Hall density is nonmonotonic due to the anisotropy in the Fermi contour of the bands. (f) Normalized superconducting critical temperature $\left(T_{c} / T_{\mathrm{c}, \max }\right)$ as a function of $\mu$ within the single-band BCS model.

depopulated. In contrast, at the (111) interface, all three $t_{2 g}$ orbitals contribute equally to both bands, and thus the effective band masses are not different enough for correlations to induce population transfer. The nonmonotonic $n_{\text {Hall }}$ in (111) is rather the result of the greater Fermi contour curvature induced by the triangular symmetry, as compared to the square symmetry at (001).

Finally, while our model does not account for the origin of superconductivity, we attempt to estimate the superconducting critical temperature for our band structure using the single-band BCS expression, $T_{c}=$ $1.13 T_{\theta} \exp \left[-\left(1 / \rho_{2} V_{\mathrm{BCS}}\right)\right]$ [54]. Here we assume that the mobile band 2 has a higher contribution to the superconductivity, and therefore use its density of states $\left(\rho_{2}\right) . T_{\theta}$ is the Debye temperature of $\mathrm{SrTiO}_{3}$ [55], and $V_{\mathrm{BCS}}$ is set so that $T_{c}$ matches the experimental value at the maximum. Figures 3(f) and 4(f) show that we get good fits for the relative positions peaks in $T_{c}$ and $n_{\text {Hall }}$ with this simplistic model.

Conclusions. - We measured the variation of quantum oscillations frequency, Hall signal, and superconducting $T_{c}$ with gate voltage in (111) $\mathrm{LaAlO}_{3} / \mathrm{SrTiO}_{3}$ and found it to be qualitatively different from the (001) interface. Employing a tight-binding model with on-site correlations, we calculated the band structure at both interfaces and showed that the difference in the crystal structure leads to bands with different orbital character. In (001) interface correlation-induced population transfer is the primary mechanism for the nonmonotonicity, while in (111) it is the shape of the symmetry-induced Fermi contours. This sets the stage for future investigation of the effect of this peculiar band structure on the superconductivity, magnetism, and ferroelectricity in these and related interfaces.

U. K. was supported by the Raymond and Beverly Sackler Faculty of Exact Sciences at Tel Aviv University and by the Raymond and Beverly Sackler Center for Computational Molecular and Material Science. Y.D. and M. G. were supported by the ISF (Grants No. 382/ 17 and 227/15), BSF (Grants No. 2014047 and 2016224), GIF (Grant No. I-1259-303.10) and the Israel Ministry of Science and Technology (Contract No. 3-12419). Part of this work was done at the High-Field Magnet Laboratory (HFML-RU/NWO), member of the European Magnetic Field Laboratory (EMFL).

U. K. and P. K. R. contributed equally to this work.

[1] A. Ohtomo and H. Y. Hwang, Nature (London) 427, 423 (2004).

[2] M. B. Shalom, M. Sachs, D. Rakhmilevitch, A. Palevski, and Y. Dagan, Phys. Rev. Lett. 104, 126802 (2010).

[3] A. D. Caviglia, S. Gariglio, C. Cancellieri, B. Sacépé, A. Fête, N. Reyren, M. Gabay, A. F. Morpurgo, and J.-M. Triscone, Phys. Rev. Lett. 105, 236802 (2010).

[4] M. B. Shalom, A. Ron, A. Palevski, and Y. Dagan, Phys. Rev. Lett. 105, 206401 (2010).

[5] E. Maniv, M. B. Shalom, A. Ron, M. Mograbi, A. Palevski, M. Goldstein, and Y. Dagan, Nat. Commun. 6, 8239 (2015).

[6] F. Trier, G. E. D. K. Prawiroatmodjo, Z. Zhong, D. V. Christensen, M. von Soosten, A. Bhowmik, J. M. GarciaLastra, Y. Chen, T. S. Jespersen, and N. Pryds, Phys. Rev. Lett. 117, 096804 (2016).

[7] A. Brinkman, M. Huijben, M. van Zalk, J. Huijben, U. Zeitler, J. C. Maan, W. G. van der Wiel, G. Rijnders, D. H. A. Blank, and H. Hilgenkamp, Nat. Mater. 6, 493 (2007).

[8] J. A. Bert, B. Kalisky, C. Bell, M. Kim, Y. Hikita, H. Y. Hwang, and K. A. Moler, Nat. Phys. 7, 767 (2011).

[9] A. Ron, E. Maniv, D. Graf, J.-H. Park, and Y. Dagan, Phys. Rev. Lett. 113, 216801 (2014).

[10] N. Reyren, S. Thiel, A. D. Caviglia, L. F. Kourkoutis, G. Hammerl, C. Richter, C. W. Schneider, T. Kopp, A.-S. Rüetschi, D. Jaccard, M. Gabay, D. A. Muller, J.-M. Triscone, and J. Mannhart, Science 317, 1196 (2007).

[11] A. D. Caviglia, S. Gariglio, N. Reyren, D. Jaccard, T. Schneider, M. Gabay, S. Thiel, G. Hammerl, J. Mannhart, and J.-M. Triscone, Nature (London) 456, 624 (2008).

[12] C. Bell, S. Harashima, Y. Kozuka, M. Kim, B. G. Kim, Y. Hikita, and H. Y. Hwang, Phys. Rev. Lett. 103, 226802 (2009).

[13] M. Salluzzo, J. C. Cezar, N. B. Brookes, V. Bisogni, G. M. De Luca, C. Richter, S. Thiel, J. Mannhart, M. Huijben, 
A. Brinkman, G. Rijnders, and G. Ghiringhelli, Phys. Rev. Lett. 102, 166804 (2009).

[14] D. Pesquera, M. Scigaj, P. Gargiani, A. Barla, J. HerreroMartín, E. Pellegrin, S. M. Valvidares, J. Gázquez, M. Varela, N. Dix, J. Fontcuberta, F. Sánchez, and G. Herranz, Phys. Rev. Lett. 113, 156802 (2014).

[15] G. Berner, M. Sing, H. Fujiwara, A. Yasui, Y. Saitoh, A. Yamasaki, Y. Nishitani, A. Sekiyama, N. Pavlenko, T. Kopp, C. Richter, J. Mannhart, S. Suga, and R. Claessen, Phys. Rev. Lett. 110, 247601 (2013).

[16] C. Cancellieri, M. L. Reinle-Schmitt, M. Kobayashi, V. N. Strocov, P. R. Willmott, D. Fontaine, P. Ghosez, A. Filippetti, P. Delugas, and V. Fiorentini, Phys. Rev. B 89, 121412(R) (2014).

[17] A. Joshua, S. Pecker, J. Ruhman, E. Altman, and S. Ilani, Nat. Commun. 3, 1129 (2012).

[18] J. Ruhman, A. Joshua, S. Ilani, and E. Altman, Phys. Rev. B 90, 125123 (2014).

[19] E. Maniv, Y. Dagan, and M. Goldstein, MRS Adv. 2, 1243 (2017).

[20] A. E. M. Smink, J. C. de Boer, M. P. Stehno, A. Brinkman, W. G. van der Wiel, and H. Hilgenkamp, Phys. Rev. Lett. 118, 106401 (2017).

[21] A. F. Santander-Syro, O. Copie, T. Kondo, F. Fortuna, S. Pailhès, R. Weht, X. G. Qiu, F. Bertran, A. Nicolaou, A. Taleb-Ibrahimi, P. Le Fèvre, G. Herranz, M. Bibes, N. Reyren, Y. Apertet, P. Lecoeur, A. Barthélémy, and M. J. Rozenberg, Nature (London) 469, 189 (2011).

[22] Z. S. Popovicć, S. Satpathy, and R. M. Martin, Phys. Rev. Lett. 101, 256801 (2008).

[23] P. Delugas, A. Filippetti, V. Fiorentini, D. I. Bilc, D. Fontaine, and P. Ghosez, Phys. Rev. Lett. 106, 166807 (2011).

[24] M. Hirayama, T. Miyake, and M. Imada, J. Phys. Soc. Jpn. 81, 084708 (2012).

[25] Z. Zhong, A. Tóth, and K. Held, Phys. Rev. B 87, 161102 (R) (2013).

[26] K. Michaeli, A. C. Potter, and P. A. Lee, Phys. Rev. Lett. 108, 117003 (2012).

[27] S. Banerjee, O. Erten, and M. Randeria, Nat. Phys. 9, 626 (2013).

[28] G. Herranz, F. Sénchez, N. Dix, M. Scigaj, and J. Fontcuberta, Sci. Rep. 2, 758 (2012).

[29] S. M. Walker, A. de la Torre, F. Y. Bruno, A. Tamai, T. K. Kim, M. Hoesch, M. Shi, M. S. Bahramy, P. D. C. King, and F. Baumberger, Phys. Rev. Lett. 113, 177601 (2014).

[30] T. C. Rödel, C. Bareille, F. Fortuna, C. Baumier, F. Bertran, P. Le Fèvre, M. Gabay, O. H. Cubelos, M. J. Rozenberg, T. Maroutian, P. Lecoeur, and A. F. Santander-Syro, Phys. Rev. Applied 1, 051002 (2014).

[31] M. S. Scheurer, D. F. Agterberg, and J. Schmalian, Quantum Mater. 2, 9 (2017).

[32] P. K. Rout, I. Agireen, E. Maniv, M. Goldstein, and Y. Dagan, Phys. Rev. B 95, 241107 (2017).
[33] P. K. Rout, E. Maniv, and Y. Dagan, Phys. Rev. Lett. 119, 237002 (2017).

[34] M. Mograbi, E. Maniv, P. K. Rout, D. Graf, J.-H. Park, and Y. Dagan, Phys. Rev. B 99, 094507 (2019).

[35] S. Davis, V. Chandrasekhar, Z. Huang, K. Han, Ariando, and T. Venkatesan, Phys. Rev. B 95, 035127 (2017).

[36] A. M. R. V. L. Monteiro, M. Vivek, D. J. Groenendijk, P. Bruneel, I. Leermakers, U. Zeitler, M. Gabay, and A. D. Caviglia, Phys. Rev. B 99, 201102(R) (2019).

[37] N. Boudjada, G. Wachtel, and A. Paramekanti, Phys. Rev. Lett. 120, 086802 (2018).

[38] S. Davis, Z. Huang, K. Han, Ariando, T. Venkatesan, and V. Chandrasekhar, Phys. Rev. B 97, 041408(R) (2018).

[39] D. Xiao, W. Zhu, Y. Ran, N. Nagaosa, and S. Okamoto, Nat. Commun. 2, 596 (2011).

[40] S. Okamoto, Phys. Rev. Lett. 110, 066403 (2013).

[41] S. Okamoto and D. Xiao, J. Phys. Soc. Jpn. 87, 041006 (2018).

[42] D. Doennig, W. E. Pickett, and R. Pentcheva, Phys. Rev. Lett. 111, 126804 (2013).

[43] J. I. Beltrán and M. C. Muñoz, Phys. Rev. B 95, 245120 (2017).

[44] G. M. De Luca, R. Di Capua, E. Di Gennaro, A. Sambri, F. M. Granozio, G. Ghiringhelli, D. Betto, C. Piamonteze, N. B. Brookes, and M. Salluzzo, Phys. Rev. B 98, 115143 (2018).

[45] See Supplemental Material at http://link.aps.org/ supplemental/10.1103/PhysRevLett.123.036805 for more details about the theoretical model and the $\mathrm{SdH}$ analysis, which includes Refs. [46-49].

[46] R. Bistritzer, G. Khalsa, and A. H. MacDonald, Phys. Rev. B 83, 115114 (2011).

[47] G. Khalsa and A. H. MacDonald, Phys. Rev. B 86, 125121 (2012).

[48] G. L. Stamokostas and G. A. Fiete, Phys. Rev. B 97, 085150 (2018).

[49] D. Shoenberg, Magnetic Oscillations in Metals (Cambridge University Press, Cambridge, England, 1984).

[50] D. I. Khomskii, Transition Metal Compounds (Cambridge University Press, Cambridge, England, 2014).

[51] Each additional layer adds new subbands and slightly modifies the structure of the lowest subband. At the densities relevant here, only the lowest subband plays an important role and we have verified that including additional layers (beyond 3) has an insignificant effect on our results [45].

[52] N. P. Ong, Phys. Rev. B 43, 193 (1991).

[53] C. M. Hurd, The Hall Effect in Metals and Alloys (Plenum Press, New York, 1972).

[54] M. Tinkham, Introduction to Superconductivity (Dover Publications, New York, 2004).

[55] G. Burns, Solid State Commun. 35, 811 (1980). 